\title{
MECHANICAL AND THERMAL PROPERTIES OF THE POLYPROPYLENE AND RICE HUSK HYBRID BIO-COMPOSITE
}

\author{
Carlos Diaz-Campoverde, Richard Pucachaqui, Orlando Taipe, \\ Patricia Proano-Sanchez \\ Department of Materials, National Polytechnic School (EPN), \\ Av. Ladron de Guevara E11-253, Quito, Ecuador.
}

\begin{abstract}
The main objective of this study is to establish the structure-properties relationship of the polypropylene-rice husk composites. The method used in this work were the experimental study of mechanical behavior, physical, thermal and microstructural characterization applied to two groups of specimens fabricated by extrusion followed by injection molding, one group using $3 \%$ by weight compatibilizer and the other without this agent, specimens were manufactured by varying the concentration of the 5-25\% reinforcement and particle size between 250-500 $\mu \mathrm{m}$. The mechanical properties were performed according to ASTM standards. Statistical analyses of the results obtained were carried out. The results show that the compatibilizer don't visibly improve the resistance to impact, density, hardness and modulus of elasticity to flexion. The increase in the reinforcement concentration exerts a positive effect on flexural strength, tensile modulus, hardness and density, and a negative effect on tensile strength and impact. Scanning electron microscopy shows a weak interface between reinforcement-matrix. From all these tests, it was determined that the structure-properties relationship don't visible improve even with the presence of the compatibilizer.
\end{abstract}

Keywords: Bio-composite, Rice husk, Polypropylene, Compatibilizer, Maleated polypropylene.

Cite this Article: Carlos Diaz-Campoverde, Richard Pucachaqui, Orlando Taipe, Patricia Proano-Sanchez, Mechanical and Thermal Properties of the Polypropylene and Rice Husk Hybrid Bio-Composite. International Journal of Mechanical Engineering and Technology. 11(6), 2020, pp. 1-10.

https://iaeme.com/Home/issue/IJMET?Volume $=11 \&$ Issue $=6$

\section{INTRODUCTION}

The increase in environmental awareness and sustainability by society and the industrial sector has made possible the investment in research and development of composite materials that have natural waste as a reinforcing element. The replacement of synthetic composite materials manufactured with synthetic reinforcement such as fiberglass, carbon fiber, Kevlar, 
clays, etc., by natural composite materials with specific characteristics and competitive mechanical properties, it is a challenge to overcome and thermoplastic polymers offer this opportunity because when combined with natural reinforcement material they have demonstrated an encouraging mechanical behavior and with prospects for improvement. This type of bio-compounds has generated a wide field of development thanks to properties such as low weight, low density, stiffness and increased mechanical properties such as tensile strength, flexion and hardness [1].

The abundant availability of natural fibers has given an impetus to the development of natural fiber composites. This development is done considering the deforestation (depletion of forest resources) with an objective of returns for the cultivation of natural fibers. Composite boards have been used in development of panel and flush doors to satisfy the low-cost housing needs. Other product development such as panel roofing sheets with sisal fibers and glass added to jute fiber produces large increase in mechanical properties of composites. Since natural fiber composite being cost-effective materials finds it application in building, construction industry (panels, false ceilings, partition boards etc.), packaging, automobile \& railway coach interiors and storage devices [2][3].

Agricultural wastes obtained from the processing of products such as wheat, barley, rye, ryegrass, rice and others, also known as lignocellulosic materials, are another form of natural materials that have now begun to be used as reinforcement material, they are cheap, easy to obtain and their processing as composite materials offer the advantage of reducing the amount of agricultural waste, they are not toxic and are basically biodegradable. This type of natural reinforcements contains a complex network of three biopolymers known as: cellulose, hemicellulose and lignin, with a high percentage of natural humidity [4]. Due to the hydrophilic properties of these materials, poor interfacial adhesion is generated between the lignocellulosic material and the hydrophobic polymer matrix such as polypropylene, causing the mechanical properties of these bio-compounds to be low [5]. In order to solve this problem, ways of modifying or treating the surface through the use of compatibilizing agents have been studied by making the polymer chains hydrophilic. The strong interfacial adhesion obtained by improving the compatibility between the hydrophilic filler and the hydrophobic matrix can improve the physical, mechanical and thermal properties of the compound [6]. In the present study, we investigate the behavior of hardness, density, fibers content and other properties of bio-compounds prepared using thermoplastic material such as matrix (polypropylene) and particulate lignocellulosic reinforcement material (rice husk), two groups of studio specimens were prepared, the first group does not contain a compatibilizing agent to the second group of specimens are added the compatibilizer (maleized polypropylene) whose concentration remains constant $(3 \%)$, the concentration $(5-25 \%)$ of reinforcement material whose added particle size is a mixture of sizes (250-500 microns).

\section{MATERIALS AND METHODS}

\subsection{Materials}

The rice husk used as reinforcement for this study corresponds to the variety (INIA 415), chemical composition is shown in Table 1. The particle size was 35-60 mesh (250-500 $\mu \mathrm{m})$. Once the reinforcement particles were obtained, they are dried in a Venticell oven at $80{ }^{\circ} \mathrm{C}$ for 24 hours to keep the humidity below $4 \%$ prior to extrusion. The material used as the matrix was a thermoplastic polypropylene polymer (PH1310) in the form of homopolymer pellets, density $0.905+/-0.005 \mathrm{~g} / \mathrm{cm}^{3}$ and melt flow index of $13 \mathrm{~g} / 10 \mathrm{~min}\left(230^{\circ} \mathrm{C} / 2160 \mathrm{~g}\right)$. The compatibilizing agent was Fusabond P613 maleized polypropylene (MAPP) with a density of $0.903 \mathrm{~g} / \mathrm{cm}^{3}$ and melt flow index of $49 \mathrm{~g} / 10 \mathrm{~min}\left(190^{\circ} \mathrm{C} / 1 \mathrm{Kg}\right)$. 
Table 1 Chemical composition of rice husk.

\begin{tabular}{ccccc}
\hline & Cellulose & Hemicellulose & Lignin & Ash \\
\hline RH & 34.30 & 29.40 & 19.30 & 17 \\
\hline
\end{tabular}

\subsection{Composites Preparation}

To obtain bio-compound, the polypropylene in pellets was mixed with rice husk (RH) in an XINDA PSHJ-35 extruder (twin-screw, 7 heating zones), the temperature profile used was $165,170,175,180,185,190,195{ }^{\circ} \mathrm{C}$ with a rotation speed of the $45 \mathrm{rpm}$. Two types of composite material were prepared in pellets, the first specimens (RH-PP) and the second group using compatibilizer (RH-PP-MAPP). Prior to the injection molding of the obtained compounds, the pellets were dried in a Venticell oven at $80{ }^{\circ} \mathrm{C}$ for $2 \mathrm{~h}$ to keep the humidity low $(<4 \%)$. The percentages of reinforcement material $(\mathrm{RH})$, polypropylene matrix $(\mathrm{PP})$ and compatibilizer (MAPP) to obtain $80 \mathrm{~g} / \mathrm{min}$ are detailed in Table 2.

Table 2 Formulation of the rice husk reinforced composites.

\begin{tabular}{ccccc}
\hline Composite $\mathbf{N}_{\mathbf{o}}$ & PP $(\mathbf{g})$ & RH $(\mathbf{g})$ & MAPP $(\mathbf{g})$ & Particle size $(\boldsymbol{\mu m})$ \\
\hline $\mathrm{A}_{100}$ & 80 & - & - & - \\
$\mathrm{B}_{95: 5}$ & 75.95 & 4.12 & - & $250<\mathrm{X}<500$ \\
$\mathrm{C}_{85: 15}$ & 67.60 & 11.88 & - & $250<\mathrm{X}<500$ \\
$\mathrm{D}_{75: 25}$ & 60.21 & 20.12 & - & $250<\mathrm{X}<500$ \\
$\mathrm{E}_{92: 5: 3}$ & 75.92 & 4.04 & 2.5 & $250<\mathrm{X}<500$ \\
$\mathrm{~F}_{82: 15: 3}$ & 68.22 & 12.11 & 2.5 & $250<\mathrm{X}<500$ \\
$\mathrm{G}_{72: 25: 3}$ & 59.67 & 19.80 & 2.5 & $250<\mathrm{X}<500$ \\
\hline
\end{tabular}

\subsection{Test Details}

The prepared bio-compounds were subjected to tests of hardness, tension, flexion and impact. Hardness was measured with Shore D BAREISS hardness tester, according to ASTM D2240. The specimens were prepared for tension testing (speed of $50 \mathrm{~mm} / \mathrm{min}$ and $\mathrm{T}=20{ }^{\circ} \mathrm{C}$ ) and three-point bending (speed of $0.1 \mathrm{~mm} / \mathrm{min}$ and $\mathrm{T}=20{ }^{\circ} \mathrm{C}$ ) in the Universal Instron 3365 testing machine according to the standards of ASTM D638-14 and ASTM-D790-17, respectively, Figure 1. The number of specimens used per test were five.

The Izod impact test is performed under the guidelines of ASTM D256-10, the dimensions of the specimen were $63.5 \mathrm{~mm}$ long., $13.5 \mathrm{~mm}$ wide and thickness of $6.7 \mathrm{~mm}$ without notch, and the test was carried out on the TINIUS OLSEN IT 504 brand impact machine. The density determination is carried out using the Archimedes principle with five samples per bio-compound, the specimens are weighed on a SHIMADZU UW-6200H scale with precision of $0.01 \mathrm{~g}$, its volume was determined and then equation 1 is applied for its calculation.

$$
\boldsymbol{\delta}=\frac{M[g]}{V\left[\mathrm{~cm}^{3}\right]}
$$

The thermogravimetric analysis (TGA) is performed on the PYRIS 1 TGA brand thermogravimetric analyzer, the analysis is carried out from room temperature to $600{ }^{\circ} \mathrm{C}$, with a temperature variation of $10{ }^{\circ} \mathrm{C}$ per minute, under controlled atmosphere of nitrogen at a constant flow of $20.0 \mathrm{ml} / \mathrm{min}$. Fourier transform infrared spectroscopy (FTIR) is run on a JASCO FT / IR-6800 Spectrometer. SEM electron microscopy is performed on a TESCAN VEGA II. 


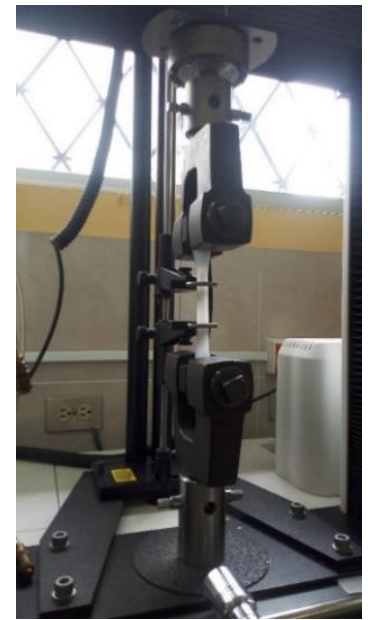

Figure 1 Universal machine for stress and flexion testing of the obtained bio-compound.

\section{RESULTS AND DISCUSSION}

\subsection{Tensile and Flexural Strength of Bio-Particulate Composites}

The mechanical properties of the bio-compound obtained with and without MAPP are shown in Table 3, the standard deviation is in brackets.

Table 3 Summary of results of tensile and flexural strength for rice husk reinforced composites.

\begin{tabular}{cccc}
\hline $\begin{array}{c}\text { Composite } \\
\text { No }\end{array}$ & $\begin{array}{c}\text { Tensile strength } \\
{[\mathbf{M P a}]}\end{array}$ & $\begin{array}{c}\text { Elongation } \\
{[\%]}\end{array}$ & $\begin{array}{c}\text { Flexural strength } \\
{[\mathbf{M P a}]}\end{array}$ \\
\hline A & $32.40(0.59)$ & $413.00(148.76)$ & $27.30(0.50)$ \\
B & $30.80(0.26)$ & $15.80(1.20)$ & $28.60(0.34)$ \\
C & $29.10(0.32)$ & $11.80(1.45)$ & $30.70(0.20)$ \\
D & $28.70(0.27)$ & $7.93(0.66)$ & $33.00(0.30)$ \\
E & $33.20(0.42)$ & $16.60(1.21)$ & $30.90(0.63)$ \\
F & $32.50(0.23)$ & $9.82(0.64)$ & $34.10(0.64)$ \\
G & $31.20(0.42)$ & $6.31(0.49)$ & $39.60(0.66)$ \\
\hline
\end{tabular}

The variation of the resistance of the compounds without and with MAPP are shown in Figure 2. For the bio-compounds (B, C, D, E, F and G) it is noted that when the reinforcement content increases, the tensile strength and flexion have an opposite behavior, the greater the content of the reinforcement, there is a decrease in tensile strength. The maximum value is in the bio-compound with MAPP (E) with an increase of $2.5 \%$. On the other hand, the flexural strength of the composite material does show an increase when compared to the pure polymer, its maximum value being 45\% (bio-compound $\mathrm{G}$ ). With respect to elongation, this is reduced as the content of reinforcing material is increased, which shows that the material increases its stiffness, a result similar to that observed by [7] and [8]. The decrease in tensile strength of the bio-compound is due to the fact that the particulate material added to the matrix reduces the movement of the polymer chains and, since there is no good interface between matrixparticle, the latter becomes a point of onset of failure through which the fracture moves. On the other hand, the improvement of the flexural strength of the two bio-compounds (F and $\mathrm{G})$ is improved with the increase of the reinforcement content, this effect being more visible with the use of the MAPP compatibilizer.

The addition of MAPP compatibilizer and reinforcing particles to the propylene matrix (PP) improves the tensile strength of the bio-compounds ( $\mathrm{E}, \mathrm{F}$ and $\mathrm{G}$ ) when compared to specimens ( $\mathrm{B}, \mathrm{C}$ and $\mathrm{D}$ ). The flexural strength compared to the polypropylene matrix the increase is acceptable, which shows that the nature of the interface formed between PP-RH is 
improved, this may be due to the interaction between the chemical groups of the coupling with the polypropylene chains and reinforcing material. According [7] and [9] the coupling agents are used to improve the interaction between the rice husk that is hydrophilic with a hydrophobic material such as polypropylene thereby increasing the mechanical properties. In both cases, the addition of particulate reinforcement to the polymer matrix causes it to pass from a ductile fracture behavior to a fragile type.

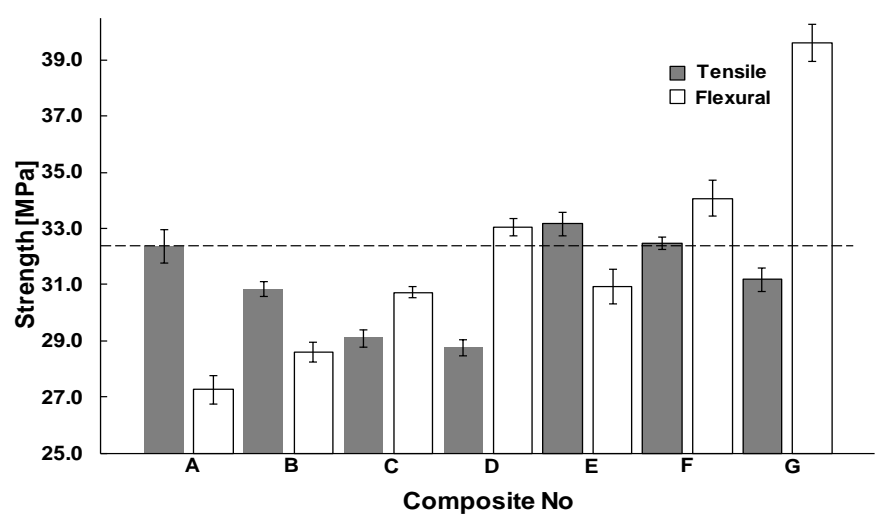

Figure 2 Tensile and flexural strength of the bio-compounds without and with MAPP.

\subsection{Tensile and Flexural Modulus of Bio-Particulate Composites}

The purpose of adding reinforcement material to the polypropylene matrix is to increase the stiffness of the resulting bio-compound. Figure 3 shows the tensile and flexural modulus obtained from the two types of bio-compound (RH-PP and RH-PP-MAPP). The tensile elasticity modulus for specimens (B, C, D, E, F and G) without and with MAPP shows an increase in its value as the content of particulate reinforcement increases in the matrix, observing a slight decrease in its value for the bio-compound $(\mathrm{E})$, there is no marked influence of the compatibilizer in terms of increasing the stiffness of the bio-compounds $(\mathrm{E}, \mathrm{F}$ and $\mathrm{G})$. The module of all bio-compounds exceeds the value of the polypropylene matrix module, with the increase for composite material (D) of $24 \%$ and, for composite material (G) with MAPP agent of 35\%. According to research carried out by [10] and [11] organic reinforcements mostly tend to improve the modulus of elasticity, while the reinforcement content increases the mechanical properties increase.

The flexural modulus according to Figure 3 does not show considerable variations in their behavior, the bio-compounds without and with MAPP with better performance (C and G) shows increases with respect to the 8 and $15 \%$ polypropylene matrix, respectively. The compatibilizing agent exerts an almost zero effect in terms of increasing the flexural stiffness of the bio-compound. [12] reports similar conclusions in their studies of polypropylene compounds and natural reinforcement.

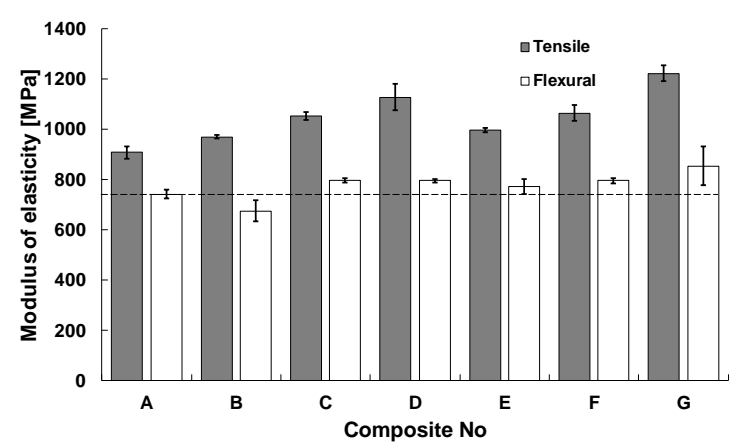

Figure 3 Behavior of flexion and tension modules of bio-composites without and with MAPP. 


\subsection{Hardness and Impact Energy Bio-Particulate Composites}

The behavior of the particulate bio-compound when varying the reinforcement content is presented in Figure 4. For the bio-compound without compatibilizer the hardness measured in specimens with different reinforcement content shows a steeply increasing slope. In those specimens that were treated with compatibilizer (MAPP), the hardness shows an increase of $2 \%$ with respect to the hardness of the samples without MAPP evidencing a low incidence of compatibilizer in this property, this slight increase may be due to a better adhesion between the fiber and the matrix, the slope obtained moves parallel to the first and with increasing tendency. The increase in the content of reinforcement particulate in the polypropylene matrix exerts a hardening effect of the matrix (for both cases), which affects the tensile strength, wear resistance, toughness among other properties.

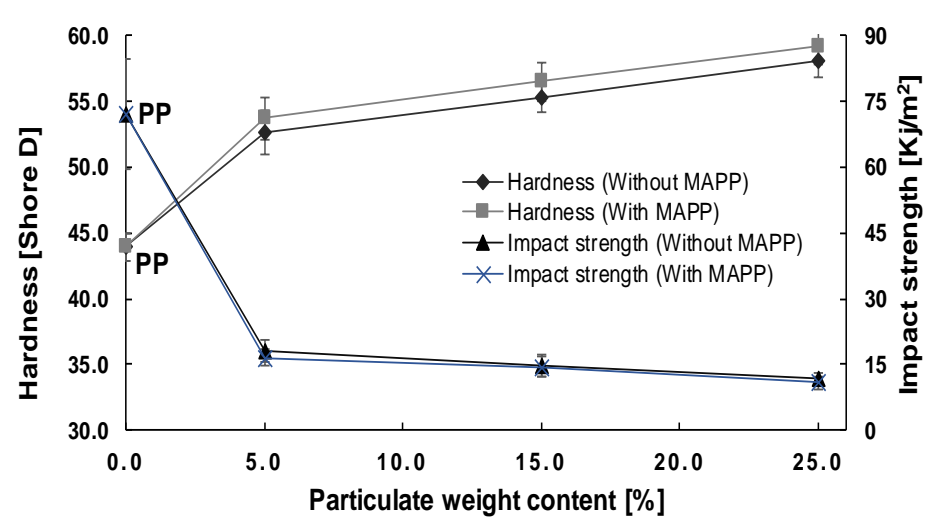

Figure 4 Hardness and impact resistance (Izod) of bio-compounds with and without MAPP.

The impact resistance shown by the two types of bio-compound does not show appreciable changes (Figure 4), its reduction with respect to the base material (PP) is approximately $75-85 \%$. The slope derived from the results obtained is of decreasing type, the influence of MAPP on the behavior of specimens is not evident. The decreasing behavior of the slope is due to the presence of the reinforcement material $(\mathrm{RH})$ in the polypropylene matrix, which would reduce the movement of the polymer chains and their lower capacity to absorb energy, a condition that facilitates the movement of the cracks through the entire matrix of polypropylene and specimen reinforcement increasing its fragility. According to studies conducted by [9] and [13], they explain that compatibilizers provide a certain degree of fragility in the final result of the biocomposites.

\subsection{Density of Bio-Particulate Composites}

Low density composite materials are ideal for those applications in which the weight is a limitation, in this study the density behavior by increasing the reinforcement content and adding compatibilizer to the matrix is shown in Figure 5.

For those specimens without compatibilizer, the variation in density is on average $4.4 \%$ with respect to the polypropylene matrix, the behavior of the bio-composite is a slight growth in density as the rice husk content increases. When the reinforcement content is $25 \%$, a slight decrease in density is observed although it continues to exceed the value of the polypropylene matrix, the decrease is due more to the increase in the amount of reinforcement than to a good reinforcement-matrix interaction. By adding 3\% compatibilizer to the matrix and to the particulate reinforcement, the density of the compound shows a slight increasing tendency with respect to the polypropylene matrix, its average increase being $6.7 \%$, this can be explained by a better interaction between RH-PP that reduces the presence of porosities and improves the compaction of the bio-compound. 


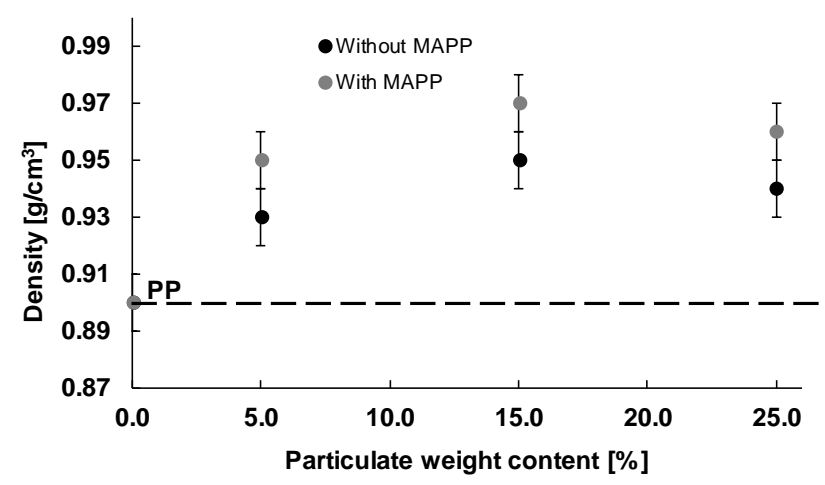

Figure 5 Density variation of bio-composites with MAPP and without MAPP.

\subsection{Thermogravimetric Analysis of Bio-Particulate Composites}

Bio-compounds B, C, D, E, F and G have a slight loss of weight and moisture, they are pyrolized in a single-stage process between $300{ }^{\circ} \mathrm{C}$ and $500{ }^{\circ} \mathrm{C}$. The weight loss is very high, this being the main stage of degradation of the structure composed of a matrix of polypropylene and particulate reinforcement, the percentage of residual matter corresponding to the ashes of the reinforcement is shown in increasing order, the lowest percentages being bio-compounds $\mathrm{B}$ and $\mathrm{E}(0.03$ and $0.02 \%)$ and the highest values for bio-compounds $\mathrm{D}$ and $\mathrm{G}$ $(0.11$ and $0.13 \%)$. For polypropylene (A) there is a loss of moisture and the beginning of pyrolysis in a single-stage process between 300 and $500{ }^{\circ} \mathrm{C}$, in this case there is no presence of residues. For the rice husk there is a loss of humidity up to $100{ }^{\circ} \mathrm{C}$, followed by two stages of degradation, the first between 250 and $450{ }^{\circ} \mathrm{C}$ and the second stage of decomposition above $450{ }^{\circ} \mathrm{C}$, Figure 6 .

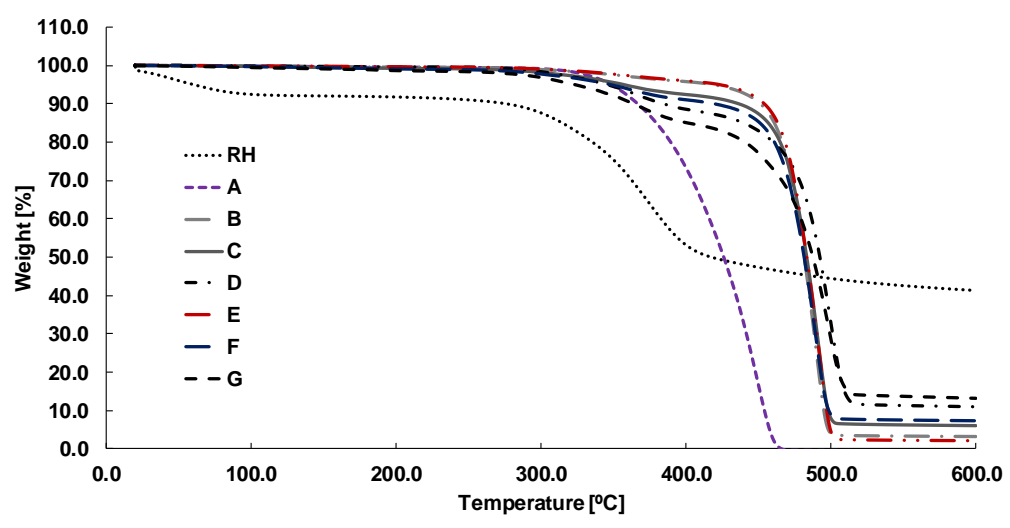

Figure 6 TGA analysis of bio-composites, matrix (PP) and rice husk (RH) reinforcement.

\subsection{Infrared Spectrographic Analysis of Bio-Particulate Composites}

The Figure 7 shows the FTIR spectra of the polypropylene matrix, rice husk and biocompound. The spectrum of the bio-compound shows the impact that rice husk particles have as reinforcement on polypropylene. The peak at $3300 \mathrm{~cm}^{-1}$ in the bio-compound represents the stretching of the $\mathrm{H}-\mathrm{OH}$ bonds. The peak that stands out in the $1600 \mathrm{~cm}^{-1}$ is typical of the lignocellulosic reinforcement and, are links of the C-O type. At $1741 \mathrm{~cm}^{-1}$ resulted from esterification reaction between free $\mathrm{OH}$ groups of the rice-husk and the functional groups of MAPP [14]. 


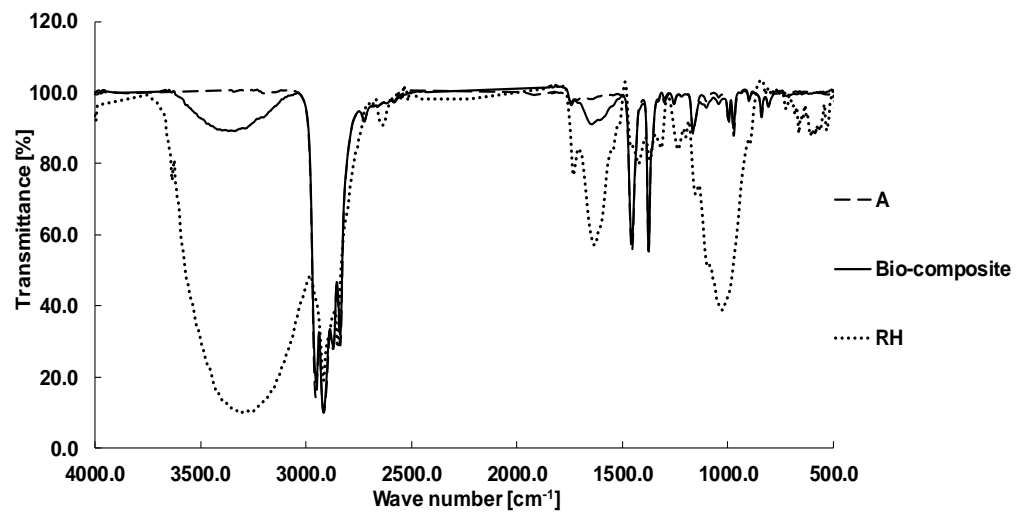

Figure 7 F-TIR matrix analysis (PP), bio-composite and rice husk (RH).

\subsection{Morphology}

The surface fractured by the stress applied to the RH-PP and RH-PP-MAPP specimens presented in Figure 8 shows the distribution of the reinforcement within the matrix. The fracture surface of the bio-compound without compatibilizing evidences the weak adhesion of the polypropylene matrix to the reinforcement $(\mathrm{RH})$, reinforcement particles separated from the matrix are shown within the formed cavities, Figure 8a.

For the bio-compound with compatibilizing (MAPP), the reinforcement (RH) has improved its adhesion with the polypropylene matrix (PP). However, there are still cavities that show the detachment of the reinforcement, the interface between matrix-reinforcement does not improve significantly, this is evidenced by the tensile strength values where the largest increase corresponds to $2.5 \%$ for compound E. In both cases Observe the fragile behavior of the bio-compounds tested, Figure $8 \mathrm{~b}$.
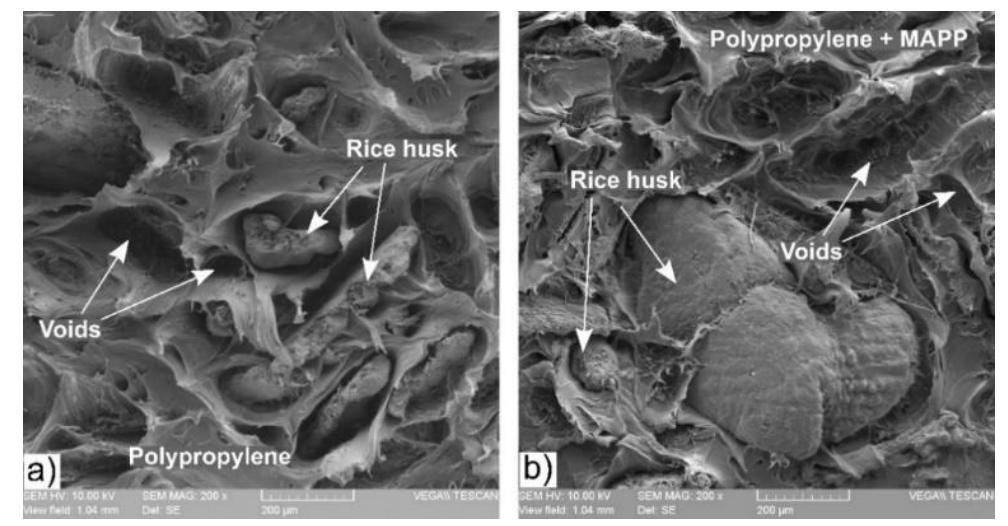

Figure 8 SEM micrograph of tensile fracture surfaces of the RH-PP and RH-PP-MAPP biocomposites: a) $25 \%$ of RH- $75 \%$ PP and b) $25 \%$ of RH-72\% PP-3\% MAPP.

\section{CONCLUSION}

The effect of the MAPP compatibilizer added to the RH-PP mixture obtained with different concentrations of reinforcement $(\mathrm{RH})$ was investigated, obtaining the following conclusions:

- By adding compatibilizer MAPP in the polypropylene matrix, the tensile and flexural strength of the bio-composites shows an improvement respect the base material.

- The increase in the concentration of reinforcement in the polypropylene matrix causes an increase in the tension module, regardless of whether or not it has compatibilizer. For its part, the flexural module shows a slight increase of up $15 \%$, no appreciable influence of the amount of reinforcement or compatibilizer is observed. 
- The hardness of the specimens without and with MAPP is proportional to the increase in reinforcement material, the hardness variation between two types of bio-compounds is $2 \%$. However, the impact resistance of the specimens is inversely proportional to the reinforcement content, reaching a minimum value when the reinforcement content is of the order of $25 \%$.

- The density of the two types of bio-compound obtained is maximum when the concentration of the reinforcement in the matrix is $15 \%$, the density variation for the bio-compounds without and with MAPP is 4.4 and 6.7\%, respectively, with respect to the polypropylene matrix.

- The addition of compatibilizer (MAPP) to the bio-compound (RH-PP) exert a determining effect on the properties of the bio-composite, the interaction between reinforcement-matrix is improved but is insufficient, and this can be observed by the presence of voids and reinforcing particles separated from the matrix shown on the SEM micrographs.

\section{ACKNOWLEDGEMENTS}

Special thanks to Departamento de Metalurgia Extractiva (DEMEX) of the Escuela Politécnica Nacional for the preparation and SEM micrographs of the specimens.

\section{REFERENCES}

[1] Bhasker Bommara, Devaiah, M., Laxmi Reddy, P., Ravindra Gandhi, M. Thermal Characterization of Fiber Reinforced Polymer Composites and Hybrid Composites, International Journal of Mechanical Engineering and Technology, 10, 2019, pp. 10551066.

[2] Ajith, G., Senthil, K., Elayaperumal, A. Experimental Investigations on Mechanical Properties of Jute Fiber Reinforced Composites with Polyester and Epoxy Resin Matrices. 12th Global Congress on Manufacturing and Management, 97, 2014, pp. 2052-2063.

[3] Sanjay, M. R., Arpitha, G. R., Laxmana Naik, L., Gopalakrishna, K., Yogewsha, B. Studies on Mechanical Properties of Banan/E-Glass Fabrics Reinforced Polyester Hybrid Composites. Journal of Materials Environment Science, 7, 2016, pp. 3179-3192.

[4] Pepijn, P. Composición Química de Diversos Materiales Lignocelulósicos de Interés Industrial y Análisis Estructural de sus Ligninas, Master. Dissertation, Universidad de Sevilla, 2010.

[5] Yang, H. S., Kim, H. J., Park, H. J., Lee, B. J. and Hwang, T. S. Effect of compatibilizing agents on rice-husk flour reinforced polypropylene composites. Composite structures, 77 , 2007, pp. 45-55.

[6] Yang, H. S., Wolcott, M. P., Kim, H. S., Kim, S. and Kim, H. J. Effect of different compatibilizing agents on the mechanical properties of lignocellulosic material filled polyethylene bio-composites. Composite structures, 79, 2007, pp. 369-375.

[7] H. S. Yang, M. P. Wolcott, H. S. Kim, S. Kim and H. J. Kim, Effect of different compatibilizing agents on the mechanical properties of lignocellulosic material filled polyethylene bio-composite, Composite Structures, 79, 2019, pp. 369-375.

[8] Nayak, N., Reddappa, H., Suresh, R. and Kumar, R. The effect of reinforcing sisal fibers on the mechanical and thermal properties of polypropylene composites. Journal of Materials Environment Science, 10, 2019, pp. 1238-1249.

[9] Panthapulakkal, S., Sain, M. and Law, S. Effect of coupling agents on rice-husk-filled HDPE extruded profiles. Polymer International, 54, 2005, pp. 137-142. 
[10] Alvarez-Castillo, A., García-Hernández, E., Domínguez-Domínguez, M. M., GranandosBaeza, J. M., Aguirre-Cruz, A., Carmona-García, R., Morales-Cepeda, A., HerreraFranco, P. J., Licea-Claveríe, A., Mendoza-Martínez, A. M. Aprovechamiento integral de los materiales. Iberoamericana de Polímeros, 13, 2012, pp. 140-150.

[11] Ndiaye, D., Diop, B., Thiandoume, C., Fall, P. A., Farota, A. K. and Tidjani, A. Morphology and Thermo Mechanical Properties of Wood/Polypropylene Composites. In: Fatih Dogan, ed., Polypropylene. Croatia: IntechOpen, 2012, pp.415-428.

[12] Zaini, M. J., Fuad, M. Y. A, Ismail, Z., Mansor, M. S. and Mustafah, J. The effect of filler content and size on the mechanical properties of polypropylene/oil palm wood flour composites. Polymer International, 40, 1996, pp. 51-55.

[13] Leal, S. M., Fonseca, E., Ferreira, C. A. and Bohrz, S. M. Studies on the properties of rice-husk-filled-PP composites-effect of maleated PP. Materials Research, 12, 2009, pp. 333-338.

[14] Kim, H. S., Lee, B. H., Choi, S. W., Kim, S. and Kim, H. J. The effect of types of maleic anhydride-grafted polypropylene (MAPP) on the interfacial adhesion properties of bioflour-filled polypropylene composites. Composites: Part A: applied science and manufacturing, 38, 2007, pp. 1473-1482. 